Exercices Exercices de rhétorique

de rhétorique $\quad 1 \mid 2013$

Sur Racine

\title{
L'art de la langue et l'art du vers chez Racine (1926)
}

\section{Karl Vossler}

Traducteur : Denise Berger

\section{(2) OpenEdition}

Journals

Édition électronique

URL : http://journals.openedition.org/rhetorique/161

DOI : 10.4000/rhetorique. 161

ISSN : 2270-6909

Éditeur

UGA Éditions/Université Grenoble Alpes

Édition imprimée

ISBN : 978-2-84310-255-4

Référence électronique

Karl Vossler, «L'art de la langue et l'art du vers chez Racine (1926) », Exercices de rhétorique [En ligne], 1 I 2013, mis en ligne le 12 novembre 2013, consulté le 12 septembre 2020. URL : http://

journals.openedition.org/rhetorique/161; DOI : https://doi.org/10.4000/rhetorique.161

Ce document a été généré automatiquement le 12 septembre 2020.

\section{cc) (i) (9)}

Les contenus de la revue Exercices de rhétorique sont mis à disposition selon les termes de la Licence Creative Commons Attribution - Pas d'Utilisation Commerciale - Partage dans les Mêmes Conditions 4.0 International. 


\title{
L'art de la langue et l'art du vers chez Racine (1926)
}

\author{
Karl Vossler \\ Traduction : Denise Berger
}

Lorsqu'un dramaturge se laisse guider par l'acteur, il tombe facilement dans un théâtre de l'improvisation et du masque. On sait que la commedia dell'arte était parvenue ainsi à un point tel que l'acteur professionnel l'emportait sur l'écrivain, le rejetait même, comme l'avait fait des siècles auparavant l'acteur professionnel des mimes et des atellanes. Dans l'improvisation, le jeu n'était fondé que sur un grossier canevas (scenario) que les acteurs imaginaient ensemble et se répartissaient. Les personnages étaient des types fixes ou des masques, et ce n'était que lors de la représentation que les acteurs y coulaient en quelque sorte les mots vivants de l'instant, avec leurs mots d'esprit, leurs gestes et leurs singeries fortuites. Distribution des tâches et jeu collectif, exercice et ambiance [stimmung], routine et verve assuraient les succès de ces jeux de virtuoses dont les dramaturges français, Molière en premier lieu, mais même Corneille, tirèrent un enseignement. On a à juste titre reproché à Molière que nombre de ses personnages ne sont pas des hommes mais des masques ou des stéréotypes, que nombre des situations qu'il expose ne sont pas le fait d'un développement psychologique, mais sont le produit du jeu de l'acteur; on lui a reproché d'avoir travaillé trop souvent en acteur, à partir de l'improvisation et pour l'instant présent. Le comique des Plaideurs de Racine ne repose-t-il pas lui aussi sur le jeu de l'acteur et, pour l'essentiel, sur les mouvements du corps, les gestes expressifs et le tempérament italien de l'acteur? Le tout n'agit-il pas à la manière du lazzo et de la facétie ennoblie littérairement, s'il se peut, et élevée aussi haut qu'on le veuille, mais qui n'en est pas moins regardée comme de la comédie? Nulle raison de se formaliser. En effet, grâce à la professionnalisation de l'acteur qui se répandait pour la première fois dans toute la France et en Europe, on prenait plaisir aux questions scéniques, à l'illusion théâtrale et à la virtuosité du jeu de l'acteur; ce plaisir ne pouvait que se communiquer au dramaturge et influencer son œuvre. L'Illusion comique de Corneille est un des meilleurs exemples de cela. On y dit : 
Ainsi tous les acteurs d'une troupe comique,

Leur poème récité, partagent leur pratique.

L'un tue, et l'autre meurt, l'autre vous fait pitié ;

Mais la scène préside à leur inimitié.

Leurs vers font leurs combats, leur mort suit leurs paroles ;

Et, sans prendre intérêt en pas un de leurs rôles,

Le traître et le trahi, le mort et le vivant,

Se trouvent à la fin amis comme devant. [V, 5, v. 1617-1624]

«La scène préside. » Cette convention théâtrale, cette attitude corporatiste de l'artiste de profession, si perceptibles dans la comédie, s'étendent, plus ou moins, à la tragédie. Là aussi, on veut des types fixes et des rôles déterminés desquels pas plus l'écrivain que l'acteur ne doivent dévier ; là aussi on veut un langage stylisé.

Achille déplairait, moins bouillant et moins prompt :

J'aime à lui voir verser des pleurs pour un affront. [...]

Qu'Agamemnon soit fier, superbe, intéressé ;

Que pour ses dieux Énée ait un respect austère.

Conservez à chacun son propre caractère. [Boileau, Art poétique, III, v. 105-107

et 110-112]

3 Tout comme les Italiens pour la comédie, les Grecs et les Romains pour la tragédie fournissent les types, les matrices, les moules. Achille, Médée, Phèdre et Néron ne sont pour l'essentiel pas autrement conditionnés et définis qu'Arlequin, Sganarelle, Matamore et Isabelle. La bienséance particulière exigée pour chacun d'eux et à laquelle ils obéissent, fixe leurs gestes, leurs mouvements et leur expression. Racine a dû tenir compte de ce schématisme : en effet, même s'il était parvenu à en détourner son public, ses acteurs ne l'auraient pas suivi. De même qu'au XIx siècle la réforme de l'orthographe française s'est heurtée à l'opposition des imprimeurs, de même sans doute, l'acteur de profession aurait refusé à Racine son aide et sa compréhension, ou du moins, sans exprimer clairement ce refus, il se serait dérobé.

Mais Racine n'a pas songé du tout à briser les toutes puissantes tables des lois scéniques et stylistiques. Il n'aurait pas songé non plus à confier à un acteur de son temps des performances auxquelles - par sa nature et sa pratique - ce dernier n'aurait pas été convenablement préparé. Il n'a pas innové pour ce qui est des traditions de l'acteur ; il n'a fait que poursuivre et conserver, et même restreint, ce qu'il a trouvé au théâtre. De là, la pauvreté, la modestie et les limites extérieures de ses moyens scéniques et linguistiques, auxquelles les adversaires du classicisme ne cessent de s'opposer et qu'ils ne cessent de moquer.

$5 \quad$ Attitude qui pourtant n'est pas le moins du monde justifiée. En effet, la pauvreté ou plutôt dirions-nous la modestie et la rigoureuse économie des moyens artistiques sont déterminés chez Racine par l'importance et la profondeur dans le domaine de l'âme. Racine et ses personnages parlent peu parce qu'ils ont beaucoup à penser et à ressentir; ils sont hésitants parce que beaucoup de choses s'agitent en eux; ils se comportent en suivant la norme, les lois et les conventions, parce qu'ils ont en eux le crime et la démesure; ils s'en tiennent à ce qui est traditionnel, parce qu'ils sont victimes des événements les plus extraordinaires; ils se maîtrisent et se contrôlent parce qu'ils se sentent instables et perdus; la maîtrise qu'ils ont d'eux-mêmes est l'ancre de salut de leur luxuria et leurs manières au goût du jour sont le revers de leur aspiration à l'au-delà et à l'éternité. Sous ces stéréotypes, il y a une immense humanité sous-jacente, simplement trop humaine. 
6 La parole est utilisée par ces hommes pour ainsi dire à l'envers. Elle est moins expression et défoulement que voile et filtre de leur âme ; elle est moins libération que retenue, souci et intensification de leurs sentiments. Au lieu de sortir de leur drame grâce à la parole, par la parole ils l'approfondissent; bien loin de convaincre leur interlocuteur, ils le ramènent davantage à lui-même. L'éloquence et la rhétorique sont plus que tout leur point faible. Comment un tel discours si réfléchi et si policé peut-il agir sur l'homme agissant, sur l'action dramatique, sinon, pour l'essentiel en l'inhibant? Loin de donner une fluidité aux événements, il les complique, les intensifie et les alourdit. Si Oreste, Mithridate, ou Phèdre ne parlaient pas, ou tenaient un autre discours, s'ils prenaient de la distance avec leurs sentiments au lieu de les examiner, de les éclairer, de les intensifier quand ils en parlent, et d'en faire un monument, ils seraient sauvés. Mais comme ils sont par nature des artistes et méditent sur leur passion avec toute l'intensité et la chaleur du mot, les événements les atteignent à grand fracas.

7 Prise pour elle-même, sans la parole, l'action se précipite d'écueil en écueil jusque dans les profondeurs; ce n'est pas un fleuve, c'est une cascade sur des escaliers qui la font écumer et ne l'arrêtent qu'en apparence. Les événements aboutissent inexorablement à une fin précoce, la plupart du temps prévisible depuis le début et inévitable, et qui est retardée artificiellement par la discussion, les malentendus, les rumeurs et le reste. Les drames de Racine ne sont en fait constitués que d'actions finales, combats mortels et exécutions qui dépendent de procès ou de décisions très anciens. Pour cette raison, justement, on ne peut pas séparer chez lui la parole des actes. S'il en était autrement, en effet, on aurait un éclair, une chute, une rupture ou rien du tout, mais pas d'action. Comme nous pouvons le voir dans l'esquisse en prose du premier acte de son Iphigénie en Tauride, dès le début Racine a considéré actes et paroles comme formant une unité. Le souci de l'expression dans les détails, ce qui pourrait à peu près être qualifié de versification et de style [Diktion], est remis à plus tard. Racine esquissait tout d'abord un canevas, un scénario, dans lequel il déterminait, acte par acte et scène par scène, la situation, le devenir et en même temps la signification, le contenu, le but des conversations et des discours qui avaient lieu à ce moment-là. "Quand il avait lié toutes les scènes entre elles, il disait: "Ma tragédie est faite" comptant le reste pour rien ", rapportait son fils. Mais ce «rien» de la touche finale, l'enduit verbal de l'édifice, devenait insensiblement, comme c'est le cas pour les vrais artistes, la chose principale ; ainsi, tout à fait de la même manière, dans les comédies improvisées réussies, tout dépend, au dernier moment, de la représentation improvisée qui lors de la préparation semblait un rien. Racine travaillait en dramaturge de naissance et en dramaturge professionnel entraîné, d'une manière ordonnée, de l'ensemble au particulier, du canevas aux ornements, à l'inverse de Victor Hugo ; ce dernier commençait par déposer quelques perles rhétoriques et lyriques dans un texte préparé à l'avance et seulement après ajoutait, tout autour du drame, comme un cadre à ces joyaux poétiques.

8 Le discours, le vers, le mot sont en fait chez Racine tout et rien. Ils sont transparents et évidents comme un rien, mais ils sont individualisés, actualisés, et, si l'on veut, improvisés grâce à la participation générale de toutes les forces, c'est-à-dire créés par une véritable représentation du drame dans l'imagination de l'écrivain.

9 Pour composer de cette manière, comme on lance un navire, comme on forge le fer rouge, il faut posséder des ressources linguistiques, avoir une formation très poussée dans les humanités et la philologie. 
Depuis son enfance, Racine avait une mémoire stupéfiante des vers et des tournures latines, grecques, italiennes, espagnoles et françaises. Dans ses lettres de jeunesse, on trouve cités parfaitement et à la bonne place Cicéron, Virgile, Ovide, Tibulle, Térence, Pétrone, Pétrarque, l'Arioste, le Tasse, Cervantès, etc. Ensuite les grands auteurs tragiques de l'Antiquité se joignent aux poètes épiques, bucoliques et idylliques. Il ne dévore pas ces auteurs, il y prend du plaisir, les déguste, et pour lui ces écrivains ne se transforment pas en un fatras de lettré. Il déteste tout autant la pédanterie philosophique que l'ignorance. Faire des citations, écrit-il à Le Vasseur, le 16 mars 1662, cela sied à un prédicateur, non à un poète. En fin de compte, il utilise toutes ces lectures, toutes ces études pour former son goût. Il lit essentiellement la Bible, les Pères de l'Église et les théologiens pour leur contenu. Il ne faut pas croire, pour cela, que ses études humanistes et philologiques ne concernent que les formes du langage. Quand il a affronté le problème de la compréhension - chez Pindare par exemple -, il a cherché spécialement à s'intéresser au sens et à l'ordre du propos et, bien après seulement, au style ou à la langue. Même dans ses tentatives de jeunesse, déjà, une image, une épithète pouvaient l'émouvoir profondément. Dans ses remarques sur les Odes olympiques de Pindare, on peut lire à propos de la septième ode : «Il appelle le soleil $\dot{\alpha}$ yvov $\theta \varepsilon o ́ v$, parce qu'il purifie tout de ces rayons ${ }^{1}$ », pensée qui, vingt-cinq ans plus tard, revient dans la tragédie de la petite-fille du Soleil. Ce qui le frappe particulièrement dans l'Odyssée, c'est toujours la relation entre l'humain et le divin. Les premiers vers qu'il juge dignes d'être transcrits sont les paroles d'un dieu :

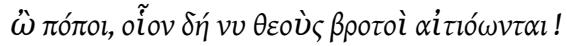

«Ah! misère !... Écoutez les mortels mettre en cause les dieux! »

C'est de nous, disent-ils, que leur viennent les maux, quand eux, en vérité,

par leur propre sottise, aggravent les malheurs assignés par leur sort ${ }^{2}$.

11 La pensée de l'homme homérique et les manières dans lesquelles elle s'exprime ne lui tiennent pas moins à cœur que la beauté ou la grâce de la forme. Par exemple : « C'est une belle chose de voir comment l'hospitalité est exercée dans l'Odyssée et la vénération avec laquelle on y reçoit tous les étrangers. » Puis il rassemble, sur cette belle pensée, exemples et expressions comme :

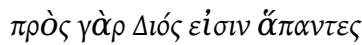

$$
\begin{aligned}
& \xi \varepsilon \tilde{v}{ }^{\prime} \text { í } \tau \varepsilon \pi \tau \omega \chi 0 i ́ \tau \varepsilon . \\
& \text { «Étrangers, mendiants, } \\
& \text { tous viennent de Zeus }{ }^{3} \text {.» }
\end{aligned}
$$

12 Ou bien : «La première chose qu'on faisait, c'étoit de boire en l'honneur des Dieux, comme de Jupiter l'Hospitalier et de quelques autres dieux, et même de ses meilleurs amis, lorsqu'ils étoient morts ou absents, comme on voit partout dans Homère et dans d'autres auteurs ${ }^{4} . »$

Au vers :

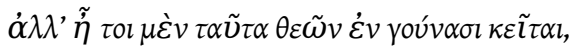

«Mais laissons tout cela sur les genoux des dieux ${ }^{5}$.»

il remarque : «Ce vers est assez fréquent dans Homère, pour marquer la providence des dieux. » La prière d'Ulysse naufragé lui fait une impression particulière :

Écoute-moi, seigneur, dont j'ignore le nom ! je viens à toi, que j'ai longtemps appelé, pour fuir hors de ces flots Poséidon et sa rage !

Les Immortels aussi n'ont-ils pas le respect d'un pauvre naufragé,

venant, comme aujourd'hui je viens à ton courant,

je viens à tes genoux, après tant d'infortunes ${ }^{6}$ ? 

affective et une résonance spirituelle. Sa fréquentation des livres est tout sauf mécanique, tout sauf formaliste, elle est tellement humaine et émotionnelle. Sa relation aux classiques est due davantage à son enfance et à sa langue maternelle qu'à la littérature. De là vient que même si le mot à mot évoque Euripide, Virgile ou Sénèque, sa poésie ne fait pas penser et même ne renvoie pas à ce qui est érudit ou étranger au français. Certains éditeurs ou commentateurs peu scrupuleux n'hésitent pas à entremêler généreusement au texte de ses drames des comparaisons avec des textes grecs, latins et français. En fait, connaissant la plupart des sources, des emprunts et des réminiscences de cet écrivain supérieur, nous ne voyons nulle part l'indication qu'il transcrit ou encore traduit, au sens philologique du mot. Mis à part Les Plaideurs - où c'est intentionnellement et en vue de l'effet comique -, ses œuvres ne contiennent aucun archaïsme savant, aucune expression spécialisée. Ce n'est que dans ses œuvres de jeunesse qu'il a beaucoup emprunté à la façon de penser et à la mode galante et précieuse. Pour le reste règne le langage courant et général de la société de cour, du commerce des lettrés et des bonnes familles, un langage châtié du cœur, de la raison et des bonnes mœurs ${ }^{12}$. Se conformer à l'usage et se maintenir modestement dans cette 
attitude est l'idéal de ce style, aspiration négative et même prosaïque. Le langage poétique de Racine ne suscite pas de grandes remarques. C'est un style sécularisé, formé par la conversation dans le monde et qui doit son élévation et sa solennité à son renoncement au sensuel, au grossier et au pittoresque. Le renoncement au plaisir des sens est le fil conducteur de sa poésie ; il en va de même pour le langage. Pour celui qui ne serait pas cultivé, cette chasteté et cette politesse sans pareilles, dans les affaires terrestres et supraterrestres, cette intériorité et cette retenue sembleraient pauvres et ennuyeuses ; pour celui qui est lettré, au contraire, elles paraissent nobles.

Dès sa jeunesse, Racine savait ce qu'il perdait et pourquoi, par ce renoncement au naturalisme et à la force du langage. À vingt-deux ans, l'Odyssée, $\mathrm{V}$, vers 234 sq., lui suggère : "Tant Homère est exact à décrire les moindres particularités : ce qui a bonne grâce dans le grec, au lieu que le latin est beaucoup plus réservé, et ne s'amuse pas à si petites choses. La langue sans doute est plus stérile, et n'a pas des mots qui expriment si heureusement les choses que la langue grecque ; car on diroit qu'il n'y a rien de bas dans le grec, et que les plus viles choses y sont noblement exprimées. Il en va de même de notre langue que de la latine: car elle fuit extrêmement de s'abaisser aux particularités, parce que les oreilles sont délicates et ne peuvent souffrir qu'on nomme des choses basses dans un discours sérieux, comme une coignée, une scie et un vilebrequin. L'italien, au contraire, ressemble au grec, et exprime tout, comme on peut voir dans l'Arioste, qui est en son genre un caractère tel celui d'Homère. » - Et de l' Odyssée, VII, v. 216 sq. : "Notre langue ne souffriroit pas dans un poème héroïque cette façon de parler, qui semble n'être propre qu'au burlesque, elle est pourtant fort ordinaire dans Homère ${ }^{13}$. » Et en X, v. 410 sq. : «Ces mots de veaux et de vaches ne sont point choquants dans le grec, comme ils le sont en notre langue, qui ne veut presque rien souffrir et qui ne souffriroit pas qu'on fit des églogues de vaches, comme Théocrite, ni qu'on parlât du porcher d'Ulysse comme d'un personnage héroïque ; mais ces délicatesse sont de véritables foiblesses. »

En accord avec ceci, Racine sentait très clairement que la phobie du quotidien qu'exprimaient le sentiment du style et le goût du langage français était une sorte de faiblesse littéraire, une préciosité, mais il ne s'y ralliait pas moins. D'emblée, peut-être simplement parce qu'il aimait le monde, la société, la mode et par sentiment national, il partageait les «faiblesses » et les délicatesses de son siècle. Il obéissait au goût de la cour, à peu près comme sa Parthénice [Stances à Parthénice, v. 12 et 15-16] :

Je me sentis esclave, et je me crus heureux

[...]

La douceur de ta voix enchanta mes oreilles;

Les nœuds de tes cheveux devinrent mes liens.

19 Mais, globalement, plus la pensée tragique de renoncement au monde au milieu même $\mathrm{du}$ monde l'envahissait, plus disparaissaient de son style la faiblesse mielleuse et l'affectation. De plus en plus ses expressions précieuses et déterminées par la mode cèdent la place à une spiritualité consciemment virile et supérieure. Sans qu'il y ait de modification remarquable de son vocabulaire ni de la construction des phrases, le style verbal de la cour prenait un aspect plus sérieux et profond grâce à la pensée tragique qui désormais le pénétrait. De La Thébaïde à Athalie, c'est pas à pas et insensiblement que s'accomplit la purification linguistique, car elle porte plus sur le contenu intellectuel que sur le corps du mot. Racine s'est peu soucié des règles grammaticales et du purisme du vocabulaire. «Écrire correctement, dit un Français d'aujourd'hui plein de goût, consiste pour nous à grouper les mots dans les phrases selon une convention 
déterminée, réduite en formules grâce à la pensée manuelle accessible à tous, et nous contraignant, par le détail des prohibitions, à demeurer dans la bonne voie. Les classiques n'usent point de cette façon uniforme d'être parfait. Il leur suffit de rester conforme à l'esprit plutôt qu'à la lettre d'une syntaxe flottante encore, et capable de recevoir, dans son indétermination relative, la marque de l'individu. Vaugelas propose plus qu'il n'impose ; le P. Bouhours ne doit légiférer que plus tard, et la législation des participes, par exemple, date de 1704. La grammaire est alors une philosophie, et non encore un code multipliant à l'infini les restrictions ${ }^{14}$.» Pour Racine, les règles d'utilisation de la langue ne sont peut-être pas une philosophie mais ressemblent à une philosophie, quelque chose qui n'a pas seulement à voir avec le bon goût mais bien plus encore avec une bonne intelligence lucide et supérieure.

La question du goût chez Racine est une question délicate et épineuse. On peut pourtant tenter de la traiter dans le cadre de l'histoire du style et pour ainsi dire la prendre au mot.

De même que la tragédie classique a dû s'élever, avec difficulté, depuis la pompe et les métamorphoses du théâtre baroque jusqu'à la simplicité, de même son langage s'est difficilement dégagé des brumes du style précieux. Le mode d'expression précieux se caractérisait par une tension exagérée, recherchée et frappante entre le sensuel et l'intellectuel. On aimait enchaîner les unes aux autres des significations qui s'opposaient par nature. On disait et on écrivait par exemple sagesse ignorante, innocemment coupable, sombrement éclairci; on aimait bien lier le grossier et le raffiné, l'expressif et l'abstrait, le naturel et l'intellectuel. Les " oreilles" devenaient «les portes de l'entendement », l'« eau » « le miroir céleste », les « yeux » « les hostelleries de la beauté ». On parlait de "cheveux d'un blond hardi», de "sécheresse de conversation ", de l'" anatomie d'un cœur ", d'une "réputation malade ", d'une «âme paralytique", etc. ${ }^{15}$ C'est en appariant d'une façon surprenante des significations hétérogènes qu'on obtenait cet effet. L'expression était étirée, tendue, tordue jusqu'au maximum et même au-dessus du signifiant et du sublime, jusqu'à éclater, jusqu'au mot d'esprit, au jeu pétillant de la raison. Aussi longtemps que ces deux mondes d'expression s'équilibrèrent, l'impression resta équivoque, le style trouble et l'on ne savait pas si c'était un héros ou un plaisantin qui parlait. Ainsi en est-il, par exemple, dans l'Agrippine de Cyrano où la veuve de Germanicus célèbre ainsi la victoire de son époux :

Déjà notre Aigle en l'air balançoit le tonnerre

Dont il devoit brûler la moitié de la terre.

Quand on vint rapporter au grand Germanicus

Qu'on voyoit l'Allemand, sous de vastes écus,

Marcher par un chemin couvert de nuit sans nombre :

«L'éclat de notre acier en dissipera l'ombre! »

Dit-il. Et pour la charge il lève le signal.

Sa voix donne la vie à des corps de métal.

Le Romain par torrens se répand dans la plaine ;

Le Colosse du Nord se soutient à grand peine :

Son énorme grandeur ne lui sert seulement

Qu'à montrer à la Parque un plus grand logement ;

Et, tandis qu'on heurtoit ces murailles humaines ;

Pour épargner le sang des Légions romaines,

Mon Héros, ennuyé du combat qui traînoit,

Se cachoit presque entier dans les coups qu'il donnoit.

Là, des bras emportés ; là des têtes brisées, etc. [La Mort d'Agrippine, I, 1, v. 17-33)] 


\section{disparaissent.}

Les palmes dont je vois ta tête si couverte

Semblent porter écrit le destin de ma perte. [Le Cid, II, 2, v. 413-414]

De la palme et de la tête a disparu le corporel, on ne voit pas des têtes recouvertes de branches de palmier. On ressent, on perçoit palme comme une valeur, la renommée, la gloire, et tête comme un chef, comme une dignité. Il ne s'agit pas d'une combinaison intellectuelle d'images mentales incompatibles, mais d'une série noble de pensées de valeur.

Cet effort sur ma flamme à mon honneur est dû. [Le Cid, III, 4, v. 924]

L'image de la flamme en relation avec des grandeurs éthiques comme l'effort et l'honneur ne peut devenir sensuelle et signifie seulement une passion enflammée. Elle devient ainsi pour sa part quelque chose d'intellectuel et d'éthique. On trouve chez Corneille un groupe de mots favoris qui, dans le contexte, perdent leur connotation première et sont élevés en quelque sorte jusqu'à l'intellectuel, le sublime et l'idéal : main, bras, fer, feu, flamme, palme, laurier, glace... Cette apothéose de la désincarnation n'est pas toujours réussie. On constate dans le style du tout jeune et du vieil écrivain des retombées peu réussies dans le terre-à-terre. Ainsi, par exemple :

Je l'ai vu tout sanglant, au milieu des batailles,

Se faire un beau rempart de mille funérailles.

ce que Corneille a eu raison de transformer en :

Je l'ai vu tout couvert de sang et de poussière

Porter partout la mort dans une armée entière. [Le Cid, I, 5, v. 277-278]

$\mathrm{Au}$ lieu de saturer notre imagination d'évidences et de la laisser se reposer dans leur contemplation, Corneille la met en action et suscite en elle une soif de supra-sensuel et d'éternel. Son style est moins rempli de sentiments qu'il n'en provoque.

Dans ce contraignant effort d'élévation, le langage de Racine plane calmement, glisse doucement et descend à proximité de la terre. Ce n'est pas que son style redevienne maintenant, soudain, images et couleurs. Il demeure dans les hauteurs, emprunte le langage de scène idéalisé de son maître et ne fait pas d'effort décisif pour restituer aux feu, flammes, fer, mains, bras, nœuds, larmes... de Corneille leur surinterprétation originelle liée aux sens. Il a laissé ce rajeunissement miraculeux aux fables divertissantes et archaïques de son ami non-tragique, La Fontaine. Lui prônait l'emploi du style tragique. C'est ainsi que l'on peut encore trouver dans les drames raciniens de l'époque tardive des vers qui, pour ce qui est du sens, ressemblent à ceux de Corneille. Par exemple :

Et sur mes foibles mains fondant leur délivrance,

Il me fit d'un empire accepter l'espérance... [Esther, I, 1, v. 51-52]

Là on ne voit pas des mains mais on songe à la vulnérabilité. Et également :

[...], le jour approche où le Dieux des armées

Va de son bras puissant faire éclater l'appui ; [...]. [Ibid., v. 20-21]

S'il y a chez Corneille des vestiges de préciosité, il y a chez Racine des vestiges de Corneille ; cependant, ils ne sont pas le trait dominant de son style. Son caractère réside moins dans la transcendance du sensuel que dans la réincarnation de la signification intellectuelle du mot. Corneille partant du mot sensuel comprime l'idée pour la faire jaillir, en quelque sorte, et s'envoler, tandis que chez Racine elle s'incline jusqu'au texte, le pénètre, le réchauffe, l'éclaire et l'élève. 
Dieu tient le cœur des rois entre ses mains puissantes. [Ibid., v. 67] dans la mesure où on la ressent religieusement. Elle provient de la Bible : «Cor regis in manu Domini » [Le cœur d'un roi est entre les mains du Seigneur] (Proverbes, XXI, 1), mais elle est rendue encore plus évocatrice et frappante par les mots tient, entre, puissante.

De mes larmes au ciel j'offrais le sacrifice. [Ibid., v. 64]

On voit la figure d'une suppliante sans que le regard s'arrête sur les détails matériels d'un sacrifice, car la tonalité affective des mots voile et transfigure l'image. Ce que nous appelons contenu d'un sentiment ne nous est signifié que par quelques traits sensuels épars mais cependant suffisants pour nous faire pressentir, nous suggérer, l'image dans sa totalité. Je cite une fois de plus la première scène d'Esther car chaque vers est propre à renforcer notre vision :

« Esther, disois-je, Esther dans la pourpre est assise,

La moitié de la terre à son sceptre est soumise,

Et de Jérusalem l'herbe cache les murs !

Sion, repaire affreux de reptiles impurs,

Voit de son temple saint les pierres dispersées,

Et du Dieu d'Israël les fêtes sont cessées ! » [Ibid., v. 83-88]

31 Est-ce que tout n'est pas vu ici du point de vue du contraste entre gloire et misère ? Esther vêtu de pourpre, dont le rouge s'enflamme un instant; le sceptre, le vent, les reptiles sollicitent l'œil intérieur pour aussitôt être comme submergés par la grande pensée de l'abandon de Dieu.

Cependant mon amour pour notre nation

A rempli ce palais de filles de Sion,

Jeunes et tendres fleurs, par le sort agitées,

Sous un ciel étranger comme moi transportées. [Ibid., v. 101-104]

Le mot « fleurs » réunit un sentiment de valeur et une image, « le sort » est à la fois le destin et vent. De même un terme abstrait comme pudeur reçoit chez Racine une coloration sensuelle :

Quelle aimable pudeur sur leur visage est peinte ! [Esther, I, 2, v. 124]

Beauté devient concret :

Ciel ! quel nombreux essaim d'innocentes beautés

S'offre à mes yeux en foule et sort de tous côtés ! [Ibid., v. 122-123]

Grâce au contexte, à l'ordre des mots, au rythme et à la sonorité, Racine sait ranimer les pauvres mots ternes.

Chez lui, la place du mot, le rythme et la rime œuvrent en commun pour instiller, dans le langage courant, vraiment fade, du personnage dramatique, la fluidité et l'harmonie. Du point de vue de l'histoire littéraire, c'est à Corneille que Racine doit ce moyen. Il le doit aussi, d'une certaine façon, à Rotrou. Il n'a nullement modifié le schéma de l'alexandrin ni l'alternance des rimes masculines et féminines. Il ne fait entendre qu'un peu plus doucement et légèrement les césures, les accents et les rimes ; d'autre part, on est un peu moins frappé par la façon dont il organise la succession des mots. Il serait peut-être exaltant de suivre dans les détails les modestes transformations et de les comparer. On vérifierait ainsi pas à pas la différence fondamentale et le changement de style entre Corneille et Racine. Ce travail minutieux conduirait à chaque fois à la même conclusion : à savoir que Racine, moins mécanique et essentiellement plus sensible que 
Corneille, utilise les possibilités offertes par le vers dramatique de son temps et par le genre artistique qui l'occupe. Pourrait-il en être autrement?

Chez Corneille, l'écrivain de la volonté responsable, on aboutit à ceci : franchissant les stades de la réflexion et de la connaissance, le héros s'élève jusqu'à la conclusion définitive ; les sentiments cessent d'être obscurs. On parvient à la lumière et l'action est désentravée. De là vient que les pauses, les accents et les rimes ont un arrière-goût intellectuel et que le discours se déroule à rebours. Des passions puissantes sont retenues, vérifiées, examinées, jusqu'à ce que la pensée parvienne à leur sommet. Il y a inversion de toute la vie intellectuelle. Il y a, semble-t-il, inhibition de la spontanéité et accélération de la conscience et de la volonté.

Les choses se passent de façon à peu près opposée chez Racine. La réflexion est trop tardive et les sentiments procèdent de façon aveugle. La parole s'écoule, les pauses, les accents, les rythmes font l'effet d'une palpitation cardiaque et donnent au vers un rythme sensuel et non intellectuel. Alors que Corneille souligne la valeur de la rime, chez Racine elle prend une résonance et une sonorité naturelles. La place du mot n'obéit plus à des décisions antérieures mais à la pression des émotions.

On a voulu expliquer cette différence de style par une attitude philosophique : Corneille aurait mis en scène l'homme cartésien et Racine celui de Pascal ou des jansénistes. En fait l'un comme l'autre ne se sont guère souciés de philosophie et ne se sont jamais et nulle part déterminés en fonction d'un système philosophique. L'homme de Descartes est essentiellement contemplatif, les héros de Corneille sont des hommes de l'action. L'homme de Pascal ou d'Arnault vit dans la théologie et la mystique, celui de Racine vit et souffre dans le monde. La littérature authentique parvient rarement du bureau ou de la cellule d'un penseur ou d'un érudit. On peut établir les contextes intellectuels grâce aux études psychologiques et à l'histoire de la culture, cela va de soi, en les trouvant chez les contemporains et les compatriotes. Nous avons nous-mêmes tissé des liens significatifs de cette nature entre les ermites de Port-Royal et les planches du théâtre racinien. Mais l'œuvre en tant que telle n'a plus rien à voir avec cela.

La tragédie de Racine mérite qu'on l'approche de toutes les façons possibles, par la critique, par l'histoire de son temps et l'histoire artistique, et que l'on fraye un accès à sa compréhension. $\mathrm{Si}$, après de tels efforts, on se trouve finalement en face d'elle, elle veut encore être entendue comme la voix d'un homme véritable, celui dont l'Iphigénie de Goethe peut dire [Iphigenie auf Tauris, I, 3, v. 452-453, "Nicht Worte sind es, die nur blenden sollen... »] :

Ces mots ne sont pas là pour t'éblouir :

J'ai découvert pour toi le tréfonds de mon cœur.

\section{NOTES}

1. CEuvres, t. VI, p. 33 [note de Vossler; c'est-à-dire renvoi à l'éd. des Grands Écrivains de la France, t. VI par P. Mesnard, Paris, Hachette, 1865, sur Gallica et archives.org ; dans l'éd. R. Picard 
de Racine, Cuvres complètes, Paris, Gallimard (Pléiade), II (Prose), 1966, p. 823, notes sur l'ode VII des Olympiques - N. d. T.].

2. Odyssée, I, v. 32 sq., traduit en allemand par Rudolf Alexander Schröder, Leipzig, 1911 [nous remplaçons par la traduction en français donnée par Victor Bérard; dans l'éd. R. Picard de Racine, p. 726].

3. Ibid., XIV, v. 57-58 [dans l'éd. R. Picard, p. 726-727].

4. « Remarques sur l'Odyssée », Euvres, t. VI, p. 60 [dans l'éd. R. Picard, p. 728].

5. [N.d.T.: I, v. 267, «Remarques sur l'Odyssée», Euvres, t. VI, p. 62-63; dans l'éd. R. Picard, p. 729.]

6. Odyssée, V, v. 445-459

7. «Remarques », p. 107 sq. [dans l'éd. R. Picard, p. 763].

8. «C'est ainsi que Racine a écrit ici.» (note de l'éd.P. Mesnard, p. 125) [dans l'éd. R. Picard, p. 776].

9. Ibid., p. 159 [dans l'éd. R. Picard, p. 801].

10. Ibid., p. 114 [dans l'éd. R. Picard, p. 768].

11. Ibid., p. 120 [dans l'éd. R. Picard, p. 772].

12. Voir les études précises « De la langue de Racine» de C.-J. Marty-Laveaux et «Étude sur le style de Racine » de P. Mesnard, CEuvres, t. VIII, p. I-XVIII et p. XIX-LXX.

13. CEuvres, t. VI, p. 102 sq. et 125 sq. [dans l'éd. R. Picard, p. 759-760 et p. 776 ; ensuite p. 804, sur Od. X, 410].

14. Truc Gonzague, Le cas Racine, Paris, Librairie Garnier frères, 1921, p. 112.

15. Voir Brunot F., Histoire de la langue française, t. III, Paris, A. Colin, 1909, p. 241 sq.

16. Bien que le cid de Corneille ait été écrit soixante-dix ans avantl'Agrippine de Cyrano de Bergerac, il marque un état plus élevé et, en un certain sens, plus tardif de l'art dramatique.

\section{AUTEURS}

\section{KARL VOSSLER}

Universitaire allemand, philologue romaniste (1872-1949) 